\title{
Effect of Allogenic Omental Graft on Esophegotomy Incision in Dogs Models
}

\author{
Hayder Hussein Abd ${ }^{1}$, Montaser Mohammede Hellal'², Ammar Maatoq Hashim³ \\ ${ }^{1}$ Research Scholar, Department of Surgery, College of veterinary medicine, Al Qassim green University, Baghdad, \\ Iraq, ${ }^{2}$ Research Scholar, Department of Surgery, College of veterinary medicine, Tikrit University, Tikrit, Iraq, \\ ${ }^{3}$ Research Scholar, Department of Surgery, College of veterinary medicine, Basrah University, Basrah, Iraq.
}

\begin{abstract}
The study aimed to evaluate the beneficial effect of allogenic omental graft on esophageal healing in dogs. Twenty healthy male adult dogs were used they were randomly divided into two equal groups (control group 10, treated group 10). Under aseptic technique $4 \mathrm{~cm}$ skin incision was made in the midline of the cervical(ventral) of animal by scalpel, after that the incision was sutured immediately by polyglycolic acid suture materials 0.3 USP used simple continous technique, and the omental graft extracted from same animals by laprotomy piece of greater omentum was then placed over the oesophagotomy incision of the experiment group and secured in place with tacks sutures, In the control group, the esophagotomy suture line was left without graft. Esophageal healing process follow up 1,2 and 3 weeks post operation by histopathological examination, the result display in treated group on the first week post operation development of collagen and lamina properia contain congested blood vessels with epithelial regeneration. 2 weeks post operation show the epithelium of the mucosa of the esophagus was formed by hyperplasia of squamous cells. The surface of these epithelia was covered by keratin with present of bundle of collagen and fibroblasts. 3 weeks post operation showed the epithelium of esophagus was covered by keratin, with the presence of blood capillaries and also, there was lymphocytic infiltration in the lamina properia with RBC. The tunica muscularis was consisting of skeletal muscle fibers, inner longitudinal, and outer circular, proliferation of fibroblast with deposition of collagen, while in control group histopathological examination in the first week show The epithelium of the esophagus was stratified squamous with the presence of keratin on its surface, 2 weeks post operation show The mucosa of the esophagus consisted of stratified squamous epithelium with a keratin layer on its surface. The lamina properia was composed of loose connective tissue with infiltration of many WBC and blood vessels, 3 weeks post operation show the basal layer of epithelium had many folds which are interdigitation with the underlying lamina properia, which appeared loose connective tissue with few numbers of fibroblasts and lymphocytes, the healing of treated group better than control group.
\end{abstract}

Keywords: Allogenic Omental Graft, Esophegotomy Incision, Dogs Models.

\section{Introduction}

Esophagectomy remains one among the foremost strict surgical procedures with considerably associated morbidity and mortality conjugation leak when esophagectomy remains a vital explanation for patient morbidity and impaired quality of life ${ }^{(14)}$. The treatment of muscle system disorders, such as, inherent anomalies and muscle system cancer, ends up in circumferential, full-thickness, extended section loss of the muscle system. Internal organ pull-up conduits or colon interpositions are wont to re-establish the sodium thiopental continuity. However, the utilization of those muscle system substitutes will cause numerous surgical morbidities and mortality ${ }^{(7,8)}$. Muscle system injuries like partial-thickness defects from membrane resections may result in pathology and refractory strictures. Similarly, full-thickness patch defects like perforations will cause leaks and fistulae. Esophagectomy finished malignant and benign conditions will cause full-thickness, longsegment, circumferential defects. These need colon or internal organ conduits to take care of sodium thiopental continuity resulting in poor quality of life ${ }^{(16)}$. However, 
involved in conjugation leak anatomic factors like a scarcity of membrane, inadequate blood offer, and fragile many factors might contribute to the high complication rate, together with lack of a membrane, the segmental nature of the blood offer, lack of peritoneum, the constant motion of swallowing and respiration and tension at the surgical web site ${ }^{(10)}$. Additionally muscle layers lying longwise that build it tough to realize watertight stitching of the esophagogastric conjugation ${ }^{(6)}$. numerous ways examining a way to stop conjugation leak are delineated antecedently. These techniques embrace buttress the conjugation with encompassing tissue like peritoneum, pleura, or pericardiac fat ${ }^{(5)}$. Therefore Augmentation of oesophagotomy web site with peritoneum, serous membrane, native muscle flap, abdomen or gut will aid healing by supporting protection and revascularizing the surgical web site ${ }^{(11)}$.

\section{Materials and Method}

Twenty male adult mixed breed dogs, 12 to 14 months old and weighing 14-18 kg, were used. Health status was determined by physical and hematological examinations. The dogs were randomly designed to two groups experimental and a control group, ten dogs in each group. All ethical considerations using animals were considered, and the experimental protocol was approved by the Ethics Committee of Basrah University. The animals were premedicated with $0.02 \mathrm{mg} / \mathrm{kg}$ atropine (KELA Laboratoria, Belgium) intramuscularly. After 10 minutes, a mixture of $5 \mathrm{mg} / \mathrm{kg}$, of 2\% Xylazine Hydrochloride (VMD-Belgium), and 15 $\mathrm{mg} / \mathrm{kg}$, of $10 \%$ Ketamine (Alfasan-Holland), were given intramuscularly as general anesthesia. After induction of anesthesia, surgical preparation was carried out in a standard method. In this study, all dogs underwent esophagotomy and laparotomy operation. To perform the cervical oesophagotomy, an incision was made at the midline on the ventral surface of the neck, beginning at the larynx and extending caudally to the manubrium. After separation and retraction of local muscles, the trachea was retracted to the right, and the cervical esophagus was exposed. After this, a stab incision was made into the lumen of the esophagus, and a 4-cm linear incision was extended longitudinally. The surgical site was rinsed using normal saline and the oesophagotomy incision was closed using 3-0 polyglycolic acid (Demetech, England) with a simple continuous pattern in both groups. To perform the laparotomy, the abdominal cavity was approached through a $7-10-\mathrm{cm}$ ventral midline incision midway between the umbilicus and pelvic inlet. A $2 \times 2$ - $\mathrm{cm} 2$ piece of the omentum was isolated by two ligatures and cut free from the remaining parts. This piece of greater omentum was then cut and placed over the oesophagotomy incision of the experiment group and secured in place with tacks sutures. In the control group, the esophagotomy suture line was left without graft, and the isolated omentum was discarded. Finally, all surgical sites were closed routinely. Postoperative management consisted of withholding of oral intake for $24 \mathrm{~h}$, and give all animals soft food during period of experiment, antibiotic (ceftiofur-Vietnam), at dose $0.1 \mathrm{mg} / \mathrm{kg}$, IM, for 3 days postoperatively).

\section{Results}

Clinical Evaluation: Based on the clinical examinations, no significant postoperative complications such as dysphasia, regurgitation, and vomiting were observed in any of the dogs during the study. All dogs had incisional swellings to some degree which resolved in a few days. All dogs were depressed and lost their appetite for $48 \mathrm{~h}$ post-surgery and regained their appetite after that. They were fed with liquid food and gruel during the first seven days after surgery and then gradually returned to their regular diet until the end of the study. All animals remained healthy throughout the study.

Macroscopic Evaluations: The macroscopic assessment of the surgical site revealed that in all dogs, the esophagus adhered to the surrounding soft tissue, and there was no noticeable difference in the extent of adhesion between the two groups. No complications such as infection, dehiscence, stricture, and fistula were observed in the surgical site of all dogs. In the experiment group, the greater omentum was completely attached to the incision site.

\section{Histopathological Results in Control Group:}

7 day: The epithelium of the esophagus was stratified squamous with the presence of keratin on its surface. The lamina properia was containing blood vessels with blood congestion surrounded by lymphocytic infiltration, which also extended along the lamina properia. The muscular coat formed by skeletal muscle fibers (fig. 1).

14 day: The mucosa of the esophagus consisted of stratified squamous epithelium with a keratin layer on its surface. The lamina properia was composed of loose connective tissue with infiltration of many WBC and blood vessels, and the submucosa was continuing with 
lamina properia. The muscular coat was skeletal muscle fibers arranged into two layers, inner longitudinal and outer circular direction. The tunica adventitia was loose connective tissue with blood vessels and wbc.(fig.2).

21 day results: The epithelium of the esophagus was stratified squamous epithelium with strands of keratin on its surface. The basal layer of epithelium had many folds which are interdigitation with the underlying lamina properia, which appeared loose connective tissue with few numbers of problasts and lymphocytes (figure 1), which these cells extended to the submucosa. The muscular layer was formed by a bundle of skeletal muscle fibers with the presence of many lymphocytes in between these muscle fibers.(Fig2, fig 3). The tunica adventitia was containing loose connective tissue fat cells and blood vessels, which appeared engorged with a moderate amount of blood.

\section{Histopathological Results in Treated Group:} The histopathological evaluation on day 7 shows the epithelium of the esophagus was stratified squamous with the presence of keratin on its surface. The keratin in certain areas was desquamated from the surface of the epithelium. The lamina properia was containing loose connective tissue with the presence of congested blood vessels, and individual WBCs were scattered in this area. The skeletal muscle fibers were encircling the esophagus. There was an area of this muscle containing atrophied skeletal muscle fiber with the presence of RBC in between skeletal muscle fibers. These skeletal muscle fibers were surrounded by collagen fibers (4).

On day 14. The epithelium of the mucosa of the esophagus was formed by hyperplasia of squamous cells which present with increasing of its thickness. The surface of these epithelia was covered by keratin with present of bundle of collagen and fibroblasts. The lamina properia was infiltrated with heavy population of lymphocytes and other wbc with many blood capillaries and larger congested blood vessels. The muscular coat was formed by skeletal muscle fibers, these was an area was devoid for these muscle but replaced by collagen fibers of skeletal muscle and many blood capillaries without blood, also present of many lymphocytes and plasma cells (fig. 5).

On day 21. The epithelium of esophagus was covered by keratin. The lamina properia was contained many congested blood vessels, with the presence of blood capillaries and newly formed vessels. Also, there was lymphocytic infiltration in the lamina properia with $\mathrm{RBC}$. The tunica muscularis was consisting of skeletal muscle fibers, inner longitudinal, and outer circular. (fig. 6).

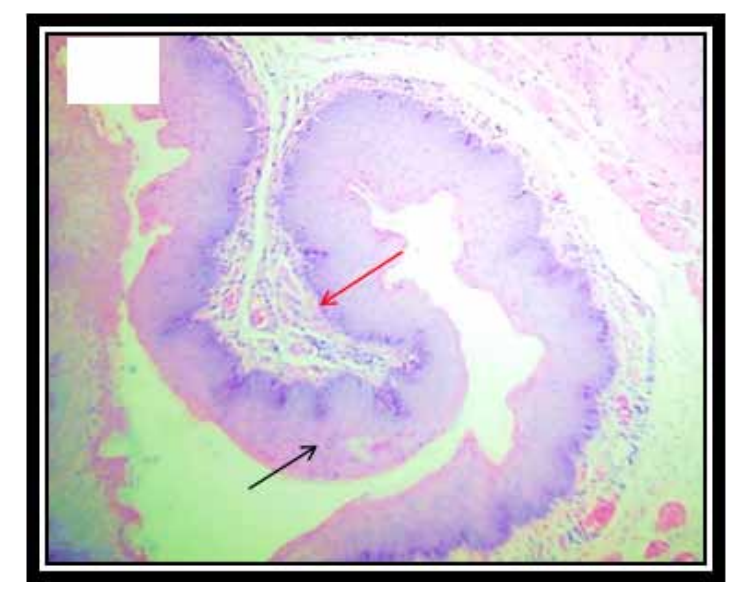

Figure 1 A Lymphocytic aggregation of lamina properia (red arrow). Congested blood vessels of esophaguse (black arrow),

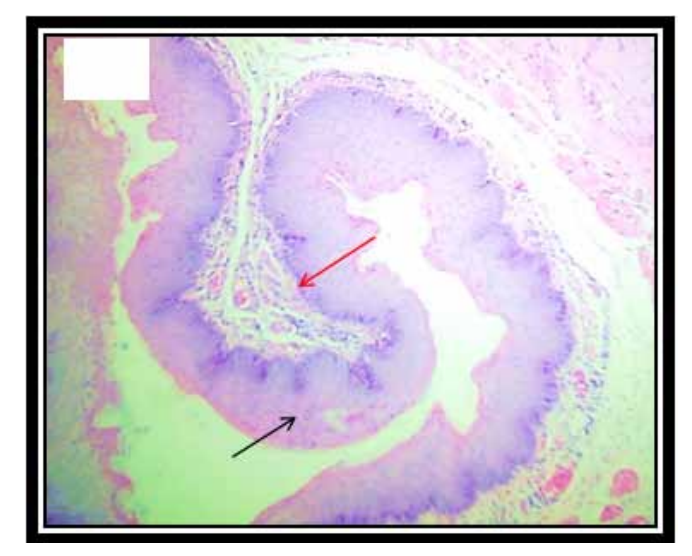

Figure 2: Stratified sequemous epithelium of esophaguse (black arrow). lamina properia (red arrow). (H\&E X100)

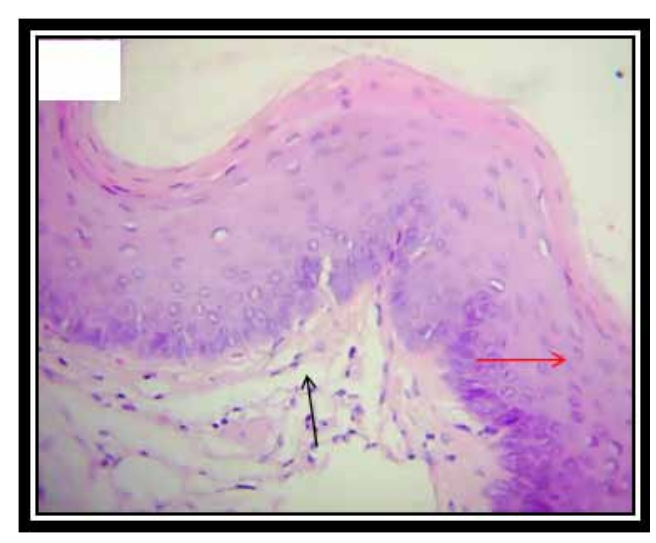

Figure 3: A. stratified sequemous epithelium (red arrow) of esophagus. Lamina properia (black arrow). 


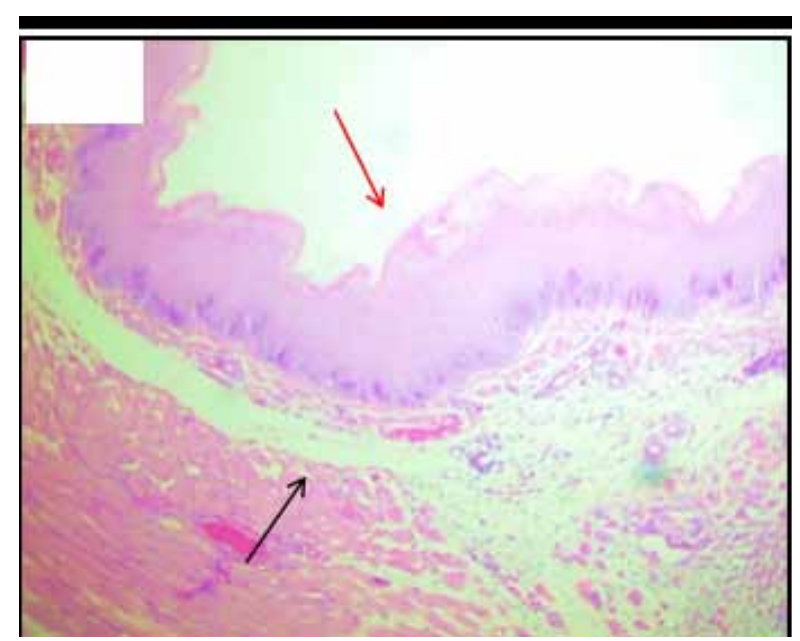

Figure 4: Stratified sequemous epithelium of esophagus (red arrow), Lamina properia (black arrow X20.

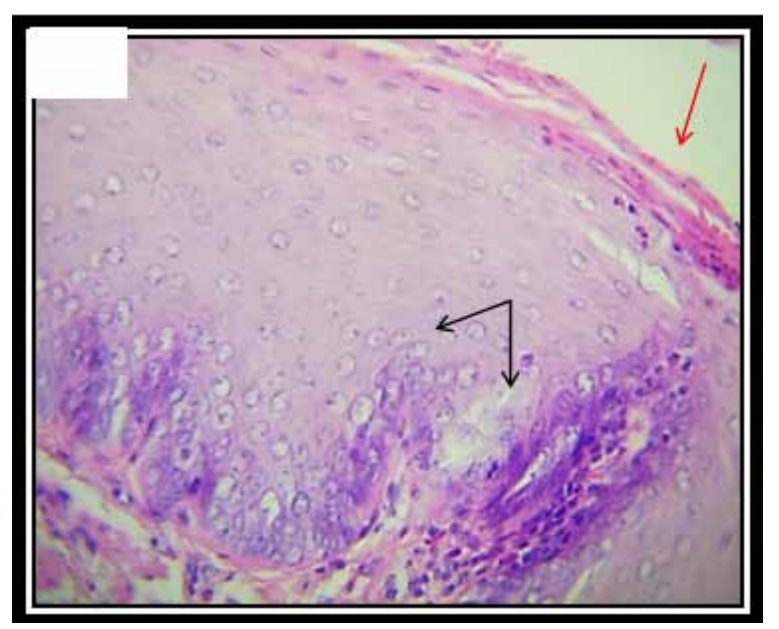

Figure 5: Hyperplasa of epithelium (black arrows), Desqumated keratin (red arrow). X40.

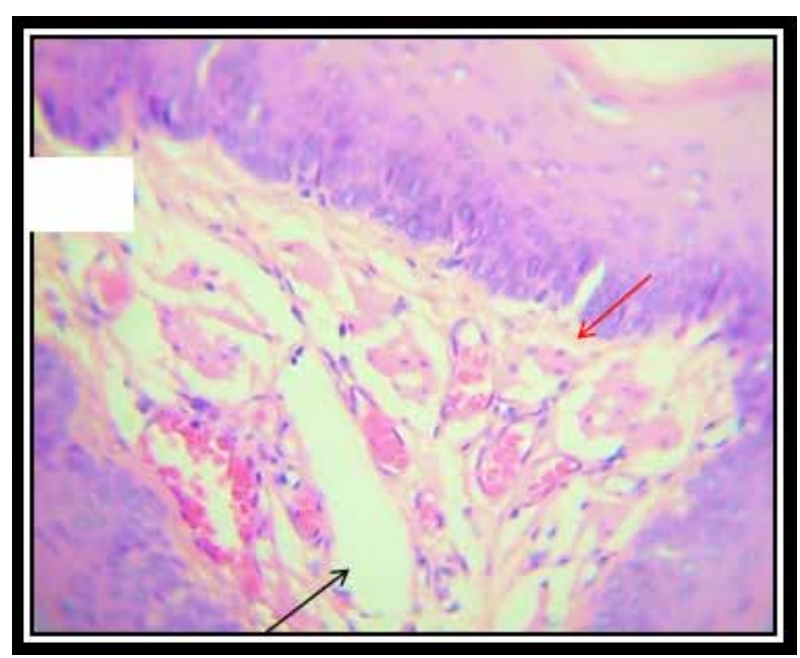

Figure 6: Stratified sequemous epithelium (red arrow), (red arrow), blood vessels congestion (red arrow). X40.

\section{Discussion}

The cervical approach was chosen in this study because we believed it is the easiest way to reach the esophagus. Trans-abdominal approach and thoracotomy in dogs are technically very difficult and accompanied by high morbidity ${ }^{[9]}$. In esophageal surgery, there is a higher risk of complications than in any other portion of the digestive tract ${ }^{[10,11]}$. One explanation could be that mesothelial cells are needed to promote anastomotic healing ${ }^{[9,12]}$. In the current study, the addition of NOA to the esophageal suture line in an extra-abdominal position might have provided the necessary mesothelial cells and consequently promote healing. Some surgeons decided to use the pedicle omentum transposition for reinforcing the anastomotic suture line to reduce the incidence of leak significantly after esophagectomy and to decrease the morbidity and mortality of the procedure ${ }^{(11)}$. The well-vascularized flap of omentum provides oxygen and nutrient for improving healing. Moreover, the omentum delivers vascular endothelial growth factor and potent angiogenic growth factor (3); this substance gives the ability to accelerate a neovascularization cross anastomosis line ${ }^{(13)}$. The exact mechanisms are not completely understood, and there is some evidence to suggest that neovascularization increases through a process that is mediated by a lipid angiogenic factor liberated by the omentum ${ }^{[14]}$. Goldsmith et al. $[15,16]$ described the angiogenic properties of the omental lipid factor and its ability to increase revascularization and neovascularization. Accordingly, in our study, it has been postulated that the transplanted NOA induced neovascularization, which is beneficial for esophageal suture line reinforcement ${ }^{[9]}$, possibly through the release of its lipid angiogenic factors (particularly vascular endothelial growth factor, VEGF). Nevertheless, it should be noted that we did not use more rigorous, accurate method, such as immunohistochemistry, to quantify neovascularization. Another possible role for the transplanted NOA in our study might be related to its mechanical function. As a matter of fact, an explanation for the impaired healing of cervical esophagotomies is its close proximity to another fresh wound. Collagenase levels might be higher in such an environment leading to the weaker suture line and, therefore, leakage [9]. Hence, we assume that NOA, as a mechanical covering, could keep esophagotomy suture line away from more superficial fresh wounds in the region. Furthermore, it has been presumed that the transplanted NOA played another role as a space-occupying structure, exerting its enhancing function by diminishing regional dead 
space from observation of experimental animals in post-operational animals did not exhibit any signs of anastomosis leak, and when euthanized the animals to take the specimen, it showed that's all anastomotic site was normally healed without any dehiscence this study agree with ${ }^{(19)}$. Also, all animals have no adhesion and stricture this agree with (17) who showed no any adhesion or stenosis at the site of anastomosis that used omental pedicle, but its record increase in the thickness of esophagus at the anastomosis site and it related with the presence of omental pedicle. And disagree with ${ }^{(18)}$ that refer not to happen stricture in his study when used omentoplasty with diaphragmatic muscle in esophageal reconstruction. Our study 21 days post-operation histopathologically showed formation of fibrous connective tissue with infiltrated of fibroblast this agree with ${ }^{(19)}$ that refer in histopathological result showed the formation of fibrous connective tissue in adipose tissue of omentum which infiltrated by inflammatory cells at 15 days post-operation in the treated group.

Conflict of Interest: None

Funding: Self

Ethical Clearance: Not required

\section{References}

1. Donington JS. Functional conduit disorders after esophagectomy. Thor Surg Clin 2006;16:53-62

2. Flanagan, J.C., Batz, R., Saboo, S.S., Nordeck, S.M., Abbara, S., Kernstine, K., and Vasan, V. Esophagectomy and Gastric Pull-through Procedures: Surgical Techniques, Imaging Features, and Potential Complications. Radiographics 36, 107, 2016

3. Zhang QX, Magovern CJ, Mack CA, Budenbender KT, Ko W, Rosengart TK. Vascular endothelial growth factor is the major angiogenic factor in omentum: mechanism of omentum-mediated angiogenesis. J Surg Res. 1997;67:147-54.

4. Hall JC, Heel KA, Papadimitriou JM, Platell C. The pathobiology of peritonitis. Gastroenterology 1998; 114:185e 96

5. AE. Oesophagus. In: Slatter D (ed) Text book of small animal surgery, 3rd edn. Saunders, Philadelphia, 2003 pp 573-575

6. Irino, T., Tsekrekos, A., Coppola, A., Scandavini, C.M., Shetye, A., Lundell, L., and Rouvelas, I.
Long-term functional outcomes after replacement of the esophagus with gastric, colonic, or jejunal conduits: a systematic literature review. Dis Esophagus 30, 1, 2017.

7. Urschel JD. Esophagogastrostomy anastomotic leaks complicating esophagectomy: a review. Am J Surg 1995;169: 634-40

8. Di Nicola, V. Omentum a powerful biological source in regenerative surgery. Regenerative Therapy, 2019. 11, 182-191.

9. Jansen PL, Klinge U, Anurov M, et al. Surgical mesh as a scaffold for tissue regeneration in the esophagus. Eur Surg Res. 2004;36:104-111.

10. Flanders JA. Problems and complications associated with oesophageal surgery. Probl Vet Med. 1989;1:183-194.

11. Lerut T, CoosemansW, Decker G, et al. Anastomotic complications after oesophagectomy. Dig Surg. 2002;19:92-98.

12. Athar MT, Chaudhry NL, Shako RK, Khan MA. Studies on end to end colonic anastomosis in the dog: A compassion of technique. Acta Vet Hung.1996;44:349- 354.

13. Morgan E, Lima O, Goldberg M, Ferdman A, Luk S, Cooper J. Successful revascularization of totally ischemic bronchial autografts with omental pedicle flap in dogs. J Thora Cardiovasc Surg. 1982;84:204210.

14. Goldsmith, H.S.; Griffith, A.L. and Catsimpoolas, $\mathrm{N}$. Increased vascular perfusion after administration of an omental lipid fraction. Surg Gynecol Obstet. 1986. 162:579-583.

15. Goldsmith, M.S.; Griffith, A.L. and Kuperman, A. Lipid angiogenic factor from omentum. J Am Med Assoc. 1984. 252:2034-2046.

16. Goldsmith, H.S. The Omentum. Research and Clinical Applications. Berlin: Springer. 1988.

17. Zieren HU, Muller JM, Pichlmaier H. Prospective randomized study of one or two - layer anastomosis following esophageal resection and cervical esophaogastrotomy. Br J Surg.1993;80:608-611.

18. Younis, B.Y. (2002). Esophageal anastomosis comparative study with or without use omental pedicle in dog. [master's thesis]. College of vet Med, Univ of Baghdad. 